\title{
A Full-Frequency Band Matching Structure of Double-Ridge Magic T
}

\author{
Chao Yuan ${ }^{1, a}$, Yi Luo ${ }^{2, b}$, Fanfu Meng ${ }^{3, c}$ and Guowei Chen ${ }^{4, d}$
}

${ }^{1}$ University of Electronic Science and Technology of China, School of Physical Electronics, Chengdu, P. R. China

${ }^{2}$ No.399, West shu Road, Chengdu, P. R. China

${ }^{3}$ University of Electronic Science and Technology of China, School of Physical Electronics, Chengdu, P. R. China

${ }^{4}$ University of Electronic Science and Technology of China, School of Physical Electronics, Chengdu, P. R. China

a375742317@qq.com, b965660661@qq.com, c1165434414@qq.com, d120720740@qq.com

Keywords: matching structure; magic T; double-ridge waveguide; power combining/dividing Abstract. In this paper, a broadband impedance matching structure is presented. Based on it, a high-power double-ridged waveguide magic T covering the full-band of WRD $750(7.5 \sim 18 \mathrm{GHz})$ is designed. The test results are: each port's return loss below $-18 \mathrm{~dB}$, synthetic branches amplitude balance error is less than $0.2 \mathrm{~dB}$, synthetic branches isolation exceeds $-18 \mathrm{~dB}$. This magic $\mathrm{T}$ has been applied to a 8-way power combining network.

\section{Introduction}

The waveguide magic $\mathrm{T}$ has found wide application in microwave technology ,especially in millimeter wave. It can be used to realize balanced mixers, E-H tuners, frequency discriminator circuits or directional couplers due to its intrinsic power split, isolation and phase reversal characteristics [1,2].

In power combining/dividing applications, the waveguide magic $\mathrm{T}$ has some advantage comparing to E/H-plane T-junction, Riblet bridge, directional coupler as: the well consistency of amplitude and phase between each distributive branch which means that extra phase-shift structure to phase matching are unnecessary; and the isolation of distributive branch is well relatively.

Although some planar/fold magic $\mathrm{T}$ which are convenient for integration have been reported. However, those magic $\mathrm{T}$ behave a poor property compare to conventional one. Therefore, the magic $\mathrm{T}$ structure this paper presents still remains an importance method in high-performance system.

The conventional rectangle waveguide magic $\mathrm{T}$ has a narrow bandwidth due to the single mode operating frequency range of rectangle waveguide, which cause certain limits in engineering. Compared with the rectangular waveguide with the same size, the double-ridge waveguide has the maximum bandwidth of 6 frequency doubling, so the double ridge waveguide magic $\mathrm{T}$ has a great advantage in the wide band applications. Moreover, its characteristic impedance is relatively low, suitable for transition to the same axis joint. The rather exact expressions for the cutoff frequency and characteristic impedance of the ridge waveguide are given in paper [3]. However, double-ridge waveguides also have its own defects: low power capacity and high metal loss, which should be considered well in practical applications.

\section{Theory}

From the structural perspective, the magic $\mathrm{T}$ is the result of mutual coincidence between the $\mathrm{E}-\mathrm{T}$ joint and the $\mathrm{H}-\mathrm{T}$ joint. Therefore, it has both the characteristics of $\mathrm{E}-\mathrm{T}$ joint and $\mathrm{H}-\mathrm{T}$ joint. Double $\mathrm{T}$ joint is a lossless four port network. As showed in Fig.1, when the electromagnetic wave is input from port 1 , port 2 and port 3 are symmetrically output, and the port 4 is isolated; when the electromagnetic wave is input from port 3, ports 2 and port 4 anti-symmetric output, and port 1 isolation. Therefore, it is a 0 or 180 degree hybrid coupler. In an ideal case, its scattering matrix is showed in Eq. 1 [4]: 


$$
[S]=\frac{1}{\sqrt{2}}\left[\begin{array}{cccc}
0 & 0 & 1 & 1 \\
0 & 0 & -1 & 1 \\
1 & -1 & 0 & 0 \\
1 & 1 & 0 & 0
\end{array}\right]
$$

In the actual situation, due to the influence of the discontinuity of the joint, the ports can not be completely matched. So the appropriate matching components must be added to eliminate the discontinuity of the ports to achieve a complete match. This kind of matching dual $\mathrm{T}$ joint is usually called the magic $\mathrm{T}$.

As mentioned before, as long as adding matching components in the E-arm and $\mathrm{H}$-arm of the dual $\mathrm{T}$ joints to eliminate reflection, the magic $\mathrm{T}$ can be achieved. But so far, the magic $\mathrm{T}$ matching components design has no precise design formula, almost all use qualitative analysis, by virtue of experience through repeated adjustments to complete the experiment.

Because the $\mathrm{E}$ arm is in the $\mathrm{E}$ plane of the double $\mathrm{T}$ joint, and mainly electric coupling with other arm through the electric field, so lumped capacitance appears in the series equivalent circuit, thus inductance components are usually used to match for E-arm. Meanwhile, the $\mathrm{H}$ arm is in the $\mathrm{H}$ plane of the double $\mathrm{T}$ joint, mainly magnetic coupling with other arm through the magnetic field, so lumped inductance appears in the parallel equivalent circuit, thus capacitor components are usually used to match for $\mathrm{H}$-arm.

In general, the impedance matching component includes: metal round bar, metal fin, metal diaphragm, size gradient and so on. A single matching method can not meet the requirements of broadband matching, so various impedance matching elements are needed to be used together. In this paper, we use diaphragm, screw, cylinder as a combination of matching components to realize the matching of magic T. This combination of matching components is inductive to E-arm, while capacitive to H-plane. The structure of the designed magic $\mathrm{T}$ is showed in Fig. 1.

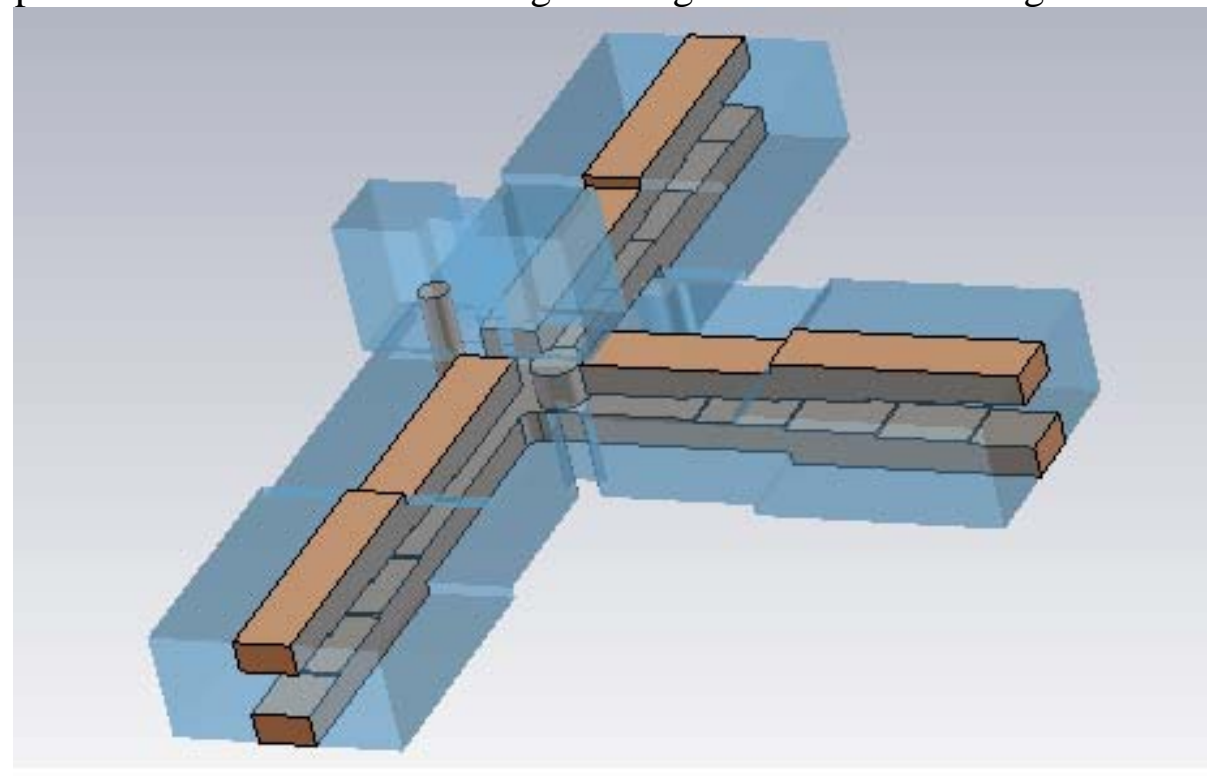

Fig. 1 Structure of magic $T$

\section{Result and Discussions}

In order to meet the broadband matching characteristics, the design of double-ridge rectangular waveguide of each port is not a standard waveguide, which is inconvenient to use in practice. So in this paper, we use the quarter-wavelength stepped impedance transformer to transform it to the WRD750 standard waveguide. The magic T is simulated with CST Microwave Studio and optimized using the efficient algorithms of Trust Region Framework. The final simulation results are showed in Fig. 2. 


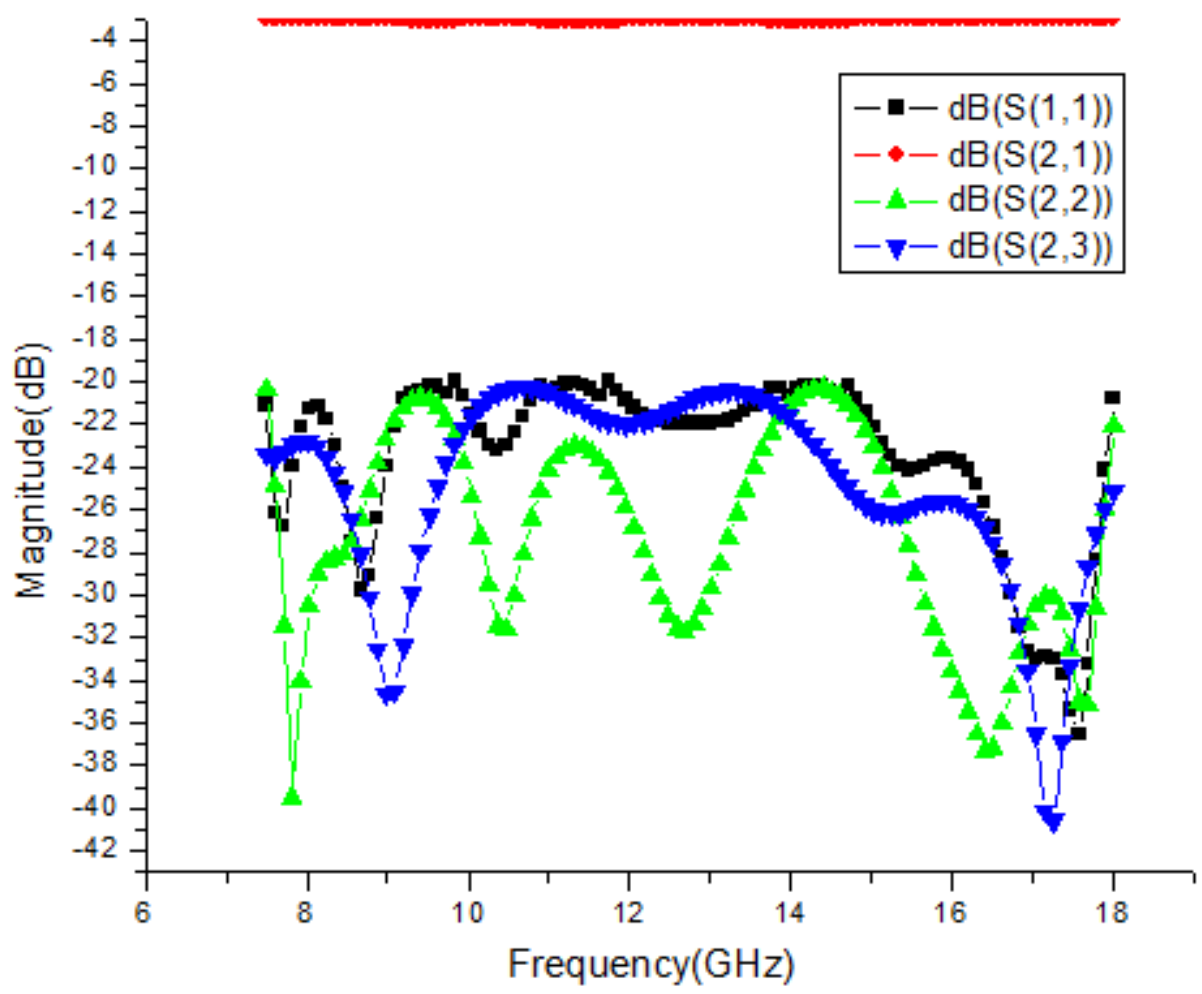

Fig. 2 Simulated scattering parameters of the magic-T

In order to minimize the influence of the ridge and the matching elements on the power capacity, the power capacity is analyzed . It is found that the position of maximum electric field intensity is located on the upper edge of the metal cylinder. After chamfered with $0.2 \mathrm{~mm}$, the power capacity has increased from 847 to $989 \mathrm{~W}$.

The practicality picture is showed in Fig. 3. In order to reduce the impact of processing precision on the performance index , the screw is set to the height adjustable. The measured result is showed in Fig. 4.

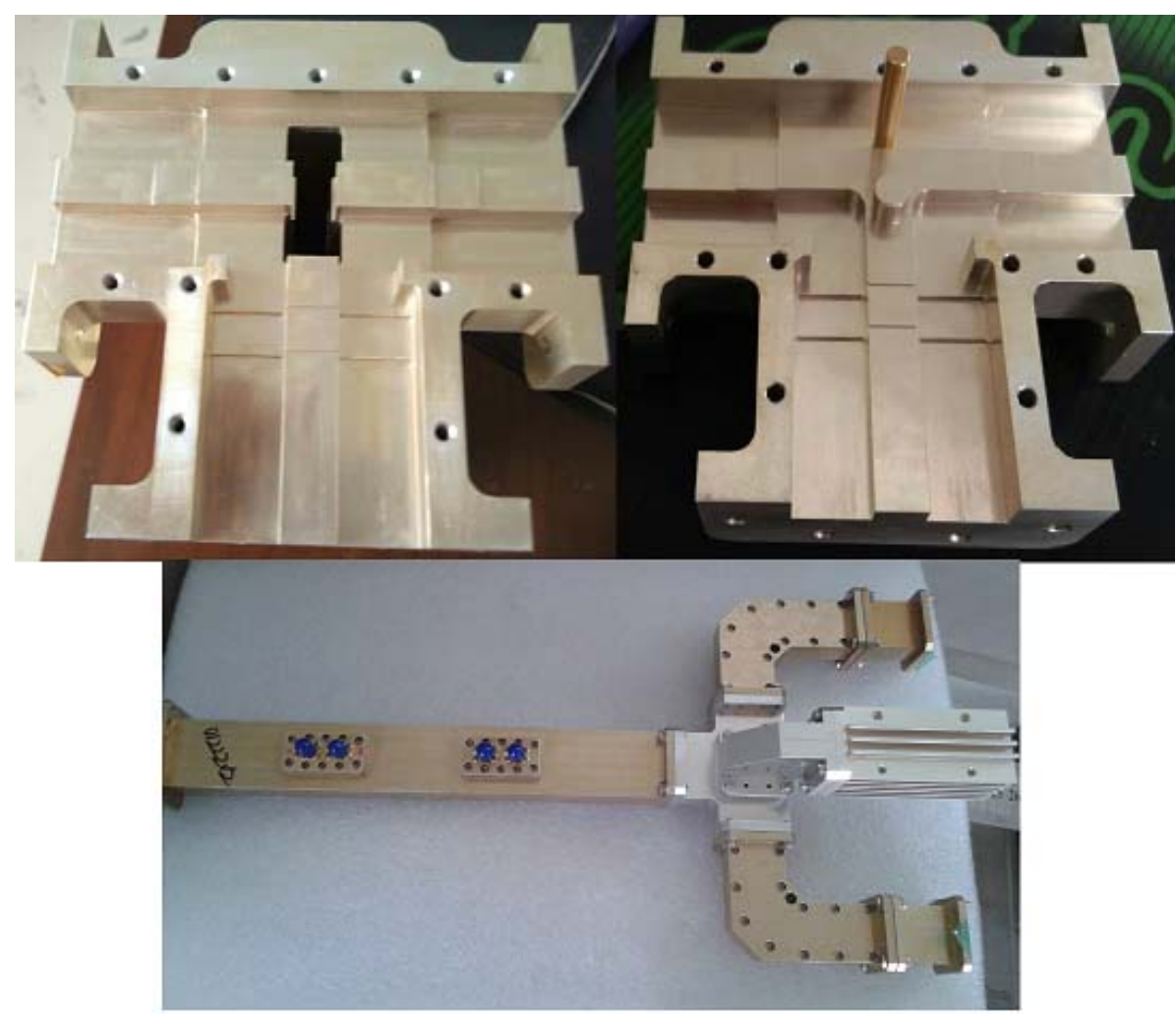

Fig. 3 Practicality picture of the magic-T 


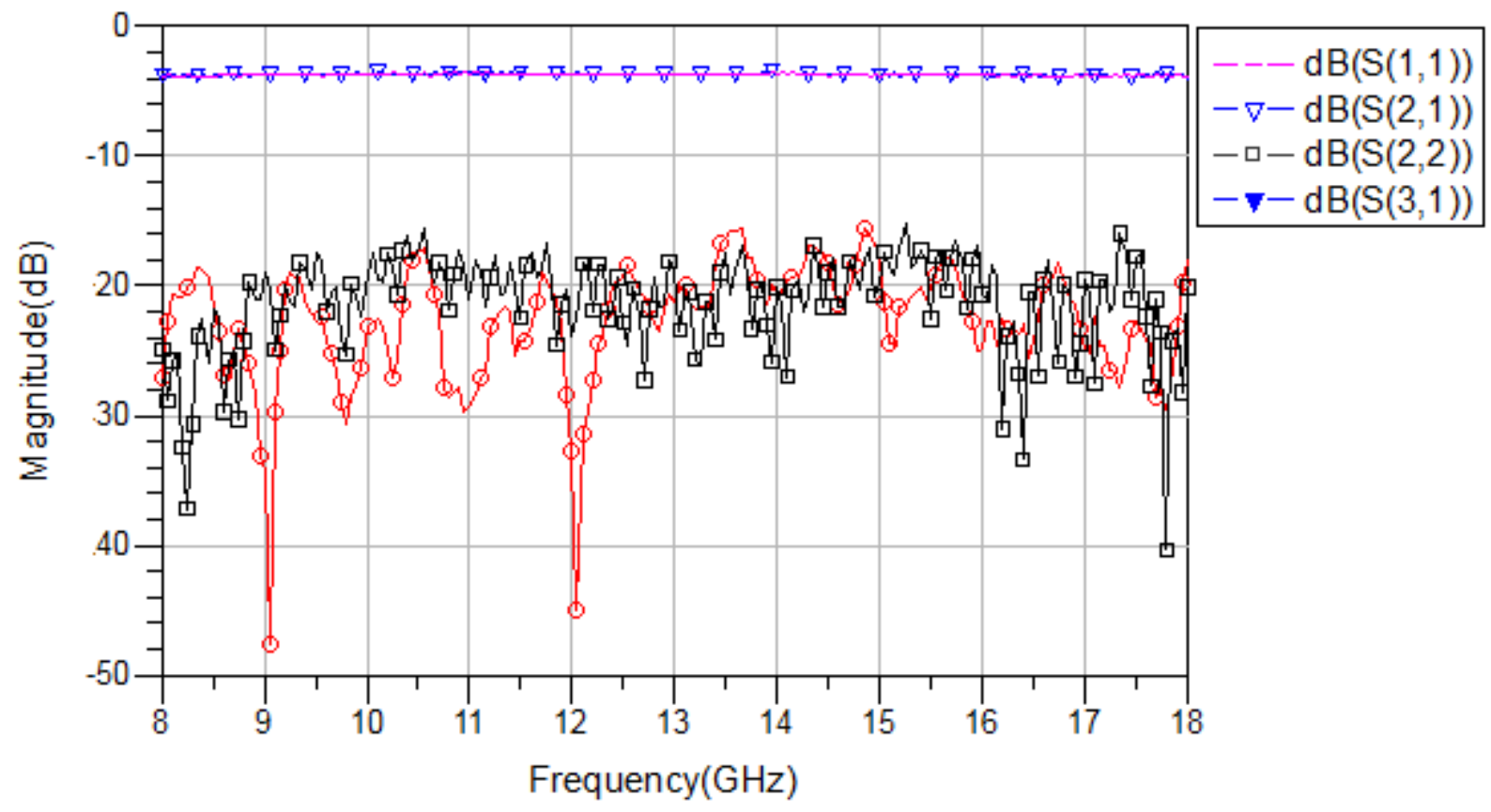

Fig. 4 Measured scattering parameters of the magic-T.

\section{Conclusions}

This letter proposes a novel wide band matching structure of double-ridge magic $\mathrm{T}$ with low loss, high isolation, and excellent phase and amplitude consistency between two output ports. Considering the loss of coaxial line to double-ridge waveguide connector, the measured results agree well with the simulated results. The load of the ridge and the matching elements in the magic $\mathrm{T}$ will reduce the power capacity, some smooth processing are necessary to improve the power capacity.

\section{Acknowledgements}

The authors would like to thank Dr. Xiaochuang Zhang, School of Physical Electronics, University of Electronic Science and Technology of China, Chengdu, China, for his valuable discussions.

\section{References}

[1] N. Marcuvitz, Waveguide Handbook. New York: McGraw-Hill, 1951.

[2] C. G. Montgomery, R. H. Dicke, and E. M. Purcell, Principles of Microwave Circuits. New York: McGraw-Hill, 1948.

[3] W. J. R. Hoefer, And Miles N. Burton, "Closed-Form Expressions For The Parameters Of Finned And Ridged Waveguides," IEEE Transactions On Microwave Theory And Techniques, Vol. MTT-30, pp. 2190-2194, Dec. 1982.

[4] D. M. Pozar, Microwave Engineering. Reading, MA: Addison-Wesley, 1990. 